$\begin{array}{cl}\text { PRAMANA } & \text { (C) Indian Academy of Sciences } \\ \begin{array}{c}\text { journal of } \\ \text { physics }\end{array} & \begin{array}{l}\text { Vol. } 69, \text { No. } 5 \\ \text { November } 2007 \\ \text { pp. } 777-782\end{array}\end{array}$

\title{
Anomalous $\boldsymbol{V} \boldsymbol{V} \boldsymbol{H}$ interactions at a linear collider
}

\author{
SUDHANSU S BISWAL ${ }^{1, *}$, DEBAJYOTI CHOUDHURY ${ }^{2}$, \\ ROHINI M GODBOLE ${ }^{1}$ and RITESH K SINGH ${ }^{3}$ \\ ${ }^{1}$ Centre for High Energy Physics, Indian Institute of Science, Bangalore 560 012, India \\ ${ }^{2}$ Department of Physics and Astrophysics, University of Delhi, New Delhi 110 007, India \\ ${ }^{3}$ Laboratoire de Physique Théoretique, 91405 Orsay Cedex, France \\ ${ }^{*}$ E-mail: sudhansu@cts.iisc.ernet.in
}

\begin{abstract}
We examine, in a model independent way, the sensitivity of a linear collider to the couplings of a light Higgs boson to a pair of gauge bosons, including the possibility of $\mathrm{CP}$ violation. We construct several observables that probe the various possible anomalous couplings. For an intermediate mass Higgs, a collider operating at a center of mass energy of $500 \mathrm{GeV}$ and with an integrated luminosity of $500 \mathrm{fb}^{-1}$ is shown to be able to constrain the $Z Z H$ vertex at the few per cent level, with even higher sensitivity for some of the couplings. However, lack of sufficient number of observables as well as contamination from the $Z Z H$ vertex limits the precision to which anomalous part of the $W W H$ coupling can be probed.
\end{abstract}

Keywords. Anomalous Higgs couplings; linear collider.

PACS Nos 13.66.Fg; 14.80.Cp; 14.70.Fm; 14.70.Hp

\section{Introduction}

The standard model (SM) of particle physics has been tested up to a high degree of accuracy, but the direct experimental verification of the phenomenon of spontaneous symmetry breaking is still pending. Various extensions of the SM have more than one Higgs boson whose CP parity and hypercharges may differ from those of the SM Higgs boson. The minimal supersymmetric standard model (MSSM) is one example of such an extended Higgs sector [1]. To establish the experimental observation of the SM Higgs boson it will be therefore, necessary to establish its properties such as hypercharge, CP parity etc. At an $e^{+} e^{-}$collider the dominant Higgs production processes are $e^{+} e^{-} \rightarrow f \bar{f} H$, which proceed via the $V V H$ coupling with $V=W, Z$ and $f$ any light fermion. Demanding Lorentz invariance, the $V V H$ couplings can be parameterized as

$$
\Gamma_{\mu \nu}=g_{V}\left[a_{V} g_{\mu \nu}+\frac{b_{V}}{m_{V}^{2}}\left(k_{1 \nu} k_{2 \mu}-g_{\mu \nu} k_{1} \cdot k_{2}\right)+\frac{\tilde{b_{V}}}{m_{V}^{2}} \epsilon_{\mu \nu \alpha \beta} k_{1}^{\alpha} k_{2}^{\beta}\right],
$$

where $k_{i}$ denote the momenta of the two $W$ 's ( $Z$ 's); $g_{W}^{\mathrm{SM}}=e \cot \theta_{W} M_{Z}$ and $g_{Z}^{\mathrm{SM}}=$ $2 e M_{Z} / \sin 2 \theta_{W}$. In general, all these anomalous couplings can be complex. For 


\section{Sudhansu S Biswal et al}

simplicity we assume $a_{V}$ to be real and close to its SM value. For processes involving $V V H$ coupling alone we can choose, without loss of generality, $g_{V}=g_{V}^{\mathrm{SM}}$ and $a_{V}=1+\Delta a_{V}$. We further assume $\Delta a_{W}=\Delta a_{Z}$ and keep terms up to linear order in the anomalous couplings. The analysis will be made for the ILC with center of mass energy $500 \mathrm{GeV}$ and a Higgs boson of mass $120 \mathrm{GeV}$. We will use $H \rightarrow$ $b \bar{b}$ final state and further assume $b$-quark detection efficiency of 0.7. The largest contribution comes from the process, $e^{+} e^{-} \rightarrow \nu_{e} \bar{\nu}_{e} H$. This process contains two missing neutrinos in the final state. However, this receives contributions from both the $W W H$ and $Z Z H$ vertices. Hence one needs to look at $e^{+} e^{-} \rightarrow Z^{*} H \rightarrow f \bar{f} H$ to constrain $Z Z H$ anomalous couplings and then make use of this information while probing $W W H$ couplings.

\section{Observables and kinematical cuts}

We have constructed various momentum combinations $\mathcal{C}_{i}$ by taking dot and scalar triple products of different linear combinations of momenta. These combinations have been listed in table 1 with their transformation properties under discrete symmetries $\mathrm{C}, \mathrm{P}$ and $\tilde{\mathrm{T}}$, where the pseudotime reversal operator $(\tilde{\mathrm{T}})$ reverses the momenta and spins of particles without interchanging their initial and final states. Then we construct observables $\left(\mathcal{O}_{i}\right)$ by taking the expectation values of the signs of various $\mathcal{C}_{i}$ 's, i.e. $\mathcal{O}_{i}=\left\langle\operatorname{sign}\left(C_{i}\right)\right\rangle$. Most of these observables have definite $\mathrm{CP}$ and $\tilde{\mathrm{T}}$ properties and hence can be used directly to probe the anomalous coupling which has the same $\mathrm{CP}$ and $\tilde{\mathrm{T}}$ properties. In our analysis we keep the terms only upto linear order in anomalous couplings $\mathcal{B}_{i}$. So all observables can be written down as

$$
\mathcal{O}\left(\left\{\mathcal{B}_{i}\right\}\right)=\sum O_{i} \mathcal{B}_{i}
$$

Measurements of these observables may be used to constrain the anomalous couplings. The possible sensitivity of these observables to the different anomalous couplings $\mathcal{B}_{i}$, at a given degree of statistical significance $f$, can be obtained by demanding $\left|\mathcal{O}\left(\left\{\mathcal{B}_{i}\right\}\right)-\mathcal{O}(\{0\})\right| \leq f \delta \mathcal{O}$. Here $\mathcal{O}(\{0\})$ is the SM value of $\mathcal{O}$ and $\delta \mathcal{O}$ is the statistical fluctuation in $\mathcal{O}$.

Table 1. List of momentum correlators, their discrete transformation properties and anomalous couplings they probe. $\vec{P}_{e}=\vec{p}_{e^{-}}-\vec{p}_{e^{+}}, \vec{P}_{f}^{+}=\vec{p}_{f}+\vec{p}_{\bar{f}}$, $\vec{P}_{f}^{-}=\vec{p}_{f}-\vec{p}_{\bar{f}}$.

\begin{tabular}{llcccccl}
\hline & Correlator & $\mathrm{C}$ & $\mathrm{P}$ & $\mathrm{CP}$ & $\tilde{\mathrm{T}}$ & $\mathrm{CP} \tilde{\mathrm{T}}$ & Probe of \\
\hline $\mathcal{C}_{0}$ & 1 & + & + & + & + & + & $a_{V}, \Re\left(b_{V}\right)$ \\
$\mathcal{C}_{1}$ & $\vec{P}_{e} \cdot \vec{P}_{f}^{+}$ & - & + & - & + & - & $\Im\left(\tilde{b}_{V}\right)$ \\
$\mathcal{C}_{2}$ & {$\left[\vec{P}_{e} \times \vec{P}_{f}^{+}\right] \cdot \vec{P}_{f}^{-}$} & + & - & - & - & + & $\Re\left(\tilde{b}_{V}\right)$ \\
$\mathcal{C}_{3}$ & {$\left[\left[\vec{P}_{e} \times \vec{P}_{f}^{+}\right] \cdot \vec{P}_{f}^{-}\right]\left[\vec{P}_{e} \cdot \vec{P}_{f}^{+}\right]$} & - & - & + & - & - & $\Im\left(b_{V}\right)$ \\
$\mathcal{C}_{4}$ & {$\left[\left[\vec{P}_{e} \times \vec{P}_{f}^{+}\right] \cdot \vec{P}_{f}^{-}\right]\left[\vec{P}_{e} \cdot \vec{P}_{f}\right]$} & $\times$ & - & $\times$ & - & $\times$ & $\Im\left(b_{V}\right), \Re\left(\tilde{b}_{V}\right)$ \\
\hline
\end{tabular}




\section{Anomalous VVH interactions}

Statistical fluctuation in cross-section and in an asymmetry can be written as

$$
\begin{aligned}
& \Delta \sigma=\sqrt{\sigma_{\mathrm{SM}} / \mathcal{L}+\epsilon^{2} \sigma_{\mathrm{SM}}^{2}}, \\
& (\Delta A)^{2}=\frac{1-A_{\mathrm{SM}}^{2}}{\sigma_{\mathrm{SM}} \mathcal{L}}+\frac{\epsilon^{2}}{2}\left(1-A_{\mathrm{SM}}^{2}\right)^{2} .
\end{aligned}
$$

Here $\sigma_{\mathrm{SM}}$ and $A_{\mathrm{SM}}$ are the SM value of cross-section and asymmetry respectively.

We choose the integrated luminosity $\mathcal{L}=500 \mathrm{fb}^{-1}$, fractional systematic error $\epsilon$ $=0.01$ and $f=3$.

Various kinematical cuts we impose, to suppress dominant background to the signal, are $5^{\circ} \leq \theta_{0} \leq 175^{\circ} ; E_{b}, E_{\bar{b}}, E_{l^{-}}, E_{l^{+}} \geq 10 \mathrm{GeV} ; p_{\mathrm{T}}^{\text {missing }} \nu \geq 15 \mathrm{GeV}$; $\Delta R_{q_{1} q_{2}} \geq 0.7 ; \Delta R_{l^{-} l^{+}} \geq 0.2 ; \Delta R_{l^{-} b}, \Delta R_{l^{-} \bar{b}}, \Delta R_{l^{+} b}, \Delta R_{l^{+} \bar{b}} \geq 0.4$.

Here $(\Delta R)^{2} \equiv(\Delta \phi)^{2}+(\Delta \eta)^{2}$ when $\Delta \phi$ and $\Delta \eta$ denote the separation between the two jets in azimuthal angle and rapidity respectively.

We additionally impose cuts on the invariant mass of the $f \bar{f}$ system:

$$
\begin{array}{ll}
R 1 \equiv\left|m_{f \bar{f}}-M_{Z}\right| \leq 5 \Gamma_{Z} \quad \text { select } Z \text {-pole, } \\
R 2 \equiv\left|m_{f \bar{f}}-M_{Z}\right| \geq 5 \Gamma_{Z} \quad \text { de-select } Z \text {-pole. }
\end{array}
$$

These enhance or suppress the contribution from $Z$ resonance in the Bjorken process respectively. $\Gamma_{Z}$ in the above is the width of $Z$ boson.

\section{3. $Z Z H$ couplings}

To probe the anomalous $Z Z H$ couplings we consider $f \bar{f}$ final state, where $f$ is any light fermion other than neutrinos. As outlined above we can construct observables with definite $\mathrm{CP}$ and $\tilde{\mathrm{T}}$ properties and thus can maximize sensitivity to the anomalous couplings for a chosen final state. One can use some of these variables to probe the anomalous couplings $[1 \mathrm{a}]$.

Cross-section: (observable $\mathcal{O}_{0}$ corresponding to correlator $\mathcal{C}_{0}$ ). Total rates are $\mathrm{CP}$ and $\tilde{\mathrm{T}}$ even quantities. Hence these can be used to constrain $\Delta a_{Z}$ and $\Re\left(b_{Z}\right)$. Total rates with $R 1$ cut and $f=\mu, u, d, c, s$ can be used to probe $\left|\Re\left(b_{Z}\right)\right|>0.48 \times 10^{-2}$. Similarly total cross-section for $f=e$ with $R 2$ cut, $\sigma(R 2 ; e)$ can probe $\Delta a_{Z}$ to $\left|\Delta a_{Z}\right|>0.038$ at $3 \sigma$ level. Figure 1a shows that the sensitivity to $\Re\left(b_{Z}\right)$ is correlated with $\Delta a_{Z}$, whereas the reverse is not true.

Forward-backward asymmetry $\left(A_{1}\right)$ : We define the FB asymmetry $A_{1}$ with respect to the polar angle of Higgs boson. Since $A_{1}$ is CP odd and $\tilde{\mathrm{T}}$ even, $A_{1}(R 1 ; \mu, q)$ can be used to probe $\Im\left(\tilde{b}_{Z}\right)$. We find that this measurement can probe $\left|\Im\left(\tilde{b}_{Z}\right)\right|>0.042$.

Up-down asymmetry $\left(A_{2}\right): A_{2}$ is the up-down asymmetry corresponding to $f$ being above or below the H-production plane. It is a CP odd and $\tilde{\mathrm{T}}$ odd observable and a real probe of $\Re\left(\tilde{b}_{Z}\right)$. Since this asymmetry requires charge determination of the final-state fermions, we cannot consider quarks in the final state. Hence using $A_{2}^{R 2}(e)$ one will be able to constrain $\left|\Re\left(\tilde{b}_{Z}\right)\right| \leq 0.064$ and it is shown by vertical lines in figure $1 \mathrm{~b}$. 
Sudhansu S Biswal et al
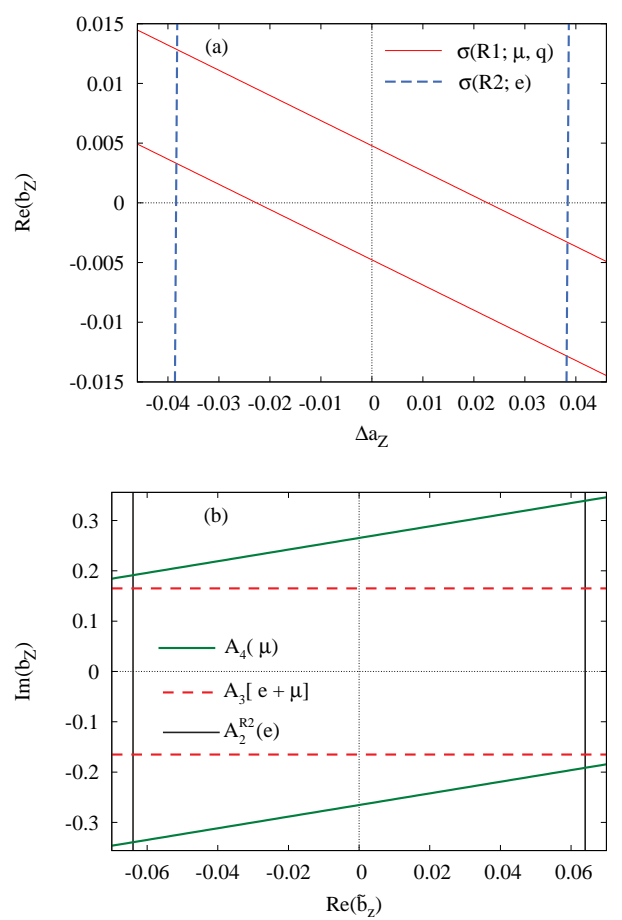

Figure 1. Simultaneous $3 \sigma$ limits on anomalous couplings with $L=500$ $\mathrm{fb}^{-1}$ : (a) $\Delta a_{Z}-\Re\left(b_{Z}\right)$ plane using cross-sections; (b) $\Re\left(\tilde{b}_{Z}\right)-\Im\left(b_{Z}\right)$ plane using various asymmetries.

Polar-azimuthal asymmetry $\left(A_{3}\right): A_{3}$ is a mixed polar-azimuthal asymmetry combining polar angle of Higgs boson and azimuthal angle of $f$ with respect to Higgs production plane and is CP even and $\tilde{\mathrm{T}}$ odd. So it is sensitive only to $\Im\left(b_{Z}\right)$. This asymmetry requires charge measurement of $f$, hence suitable only for $f=e, \mu$. This can give a sensitivity at $3 \sigma$ level as $\left|\Im\left(b_{Z}\right)\right| \leq 0.17$. The region inside the horizontal lines in figure $1 \mathrm{~b}$ shows $3 \sigma$ variation in $A_{3}$.

Another combined asymmetry $\left(A_{4}\right)$ : We construct this combined asymmetry with respect to the polar and azimuthal angles of final state $f$. Although $A_{4}$ is $\tilde{\mathrm{T}}$ odd, it does not have any definite CP property. So it is sensitive to both $\Im\left(b_{Z}\right)$ and $\Re\left(\tilde{b}_{Z}\right)$. Also $A_{4}$ requires charge determination of $f$ and hence we cannot consider quarks in the final-state for this observable. But we consider only $f=\mu$, because for $f=e$ many anomalous couplings contribute significantly with $R 1$ cut. The corresponding constraint is shown in figure $1 \mathrm{~b}$ with slant lines.

In table 2 we list all the achievable limits obtained above. We emphasize that all of them, except for $\Delta a_{Z}$ and $\Re\left(b_{Z}\right)$, are independent of other anomalous couplings. Table 2 shows that the constraint on $\Re\left(b_{Z}\right)$ depends on $\Delta a_{Z}$. Also $\tilde{T}$-odd observables require charge measurement of final-state fermions and hence quarks in the final-state cannot be considered to probe $\tilde{\mathrm{T}}$-odd couplings leading to rather poor sensitivity to them. 


\section{Anomalous VVH interactions}

Table 2. Sensitivity achievable at $3 \sigma$ level for various anomalous couplings with $L=500 \mathrm{fb}^{-1}$.

\begin{tabular}{lll}
\hline Coupling & $3 \sigma$ Bound & Observable used \\
\hline$\left|\Delta a_{Z}\right|$ & 0.038 & $\sigma$ with $R 2$ cut; $f=e^{-}$ \\
$\left|\Re\left(b_{Z}\right)\right|$ & $\left\{\begin{array}{l}0.0048\left(\Delta a_{Z}=0\right) \\
0.013\left(\left|\Delta a_{Z}\right|=0.038\right)\end{array}\right.$ & $\sigma$ with $R 1$ cut; $f=\mu, q$ \\
$\left|\Im\left(b_{Z}\right)\right|$ & 0.17 & $A_{3}$ with $R 1$ cut; $f=\mu^{-}, e^{-}$ \\
$\left|\Re\left(\tilde{b}_{Z}\right)\right|$ & 0.064 & $A_{2}\left(\phi_{e^{-}}\right)$with $R 2$ cut \\
$\left|\Im\left(\tilde{b}_{Z}\right)\right|$ & 0.042 & $A_{1}\left(c_{H}\right)$ with $R 1$ cut; $f=\mu, q$ \\
\hline
\end{tabular}

Table 3. Individual $3 \sigma$ limits of sensitivity.

\begin{tabular}{|c|c|c|c|}
\hline Coupling & & Limit & Observable used \\
\hline$|\Delta a|$ & $\leq$ & 0.018 & $\sigma_{R 2}$ \\
\hline$\left|\Re\left(b_{W}\right)\right|$ & $\leq$ & 0.098 & $\sigma_{R 2}$ \\
\hline$\left|\Im\left(b_{W}\right)\right|$ & $\leq$ & 0.62 & $\sigma_{R 1}$ \\
\hline$\left|\Re\left(\underset{\sim}{\tilde{b}_{W}}\right)\right|$ & $\leq$ & 1.6 & $A_{F B}^{1}\left(c_{H}\right)$ \\
\hline$\left|\Im\left(\tilde{b}_{W}\right)\right|$ & $\leq$ & 0.39 & $A_{F B}^{2}\left(c_{H}\right)$ \\
\hline
\end{tabular}

Table 4. Simultaneous $3 \sigma$ limits of sensitivity.

\begin{tabular}{llcl}
\hline Coupling & & $\Delta a=0$ & $\Delta a \neq 0$ \\
\hline$|\Delta a|$ & $\leq$ & - & 0.038 \\
$\left|\Re\left(b_{W}\right)\right|$ & $\leq$ & 0.10 & 0.31 \\
$\left|\Im\left(b_{W}\right)\right|$ & $\leq$ & 1.6 & 1.6 \\
$\left|\Re\left(\tilde{b}_{W}\right)\right|$ & $\leq$ & 3.2 & 3.2 \\
$\left|\Im\left(\tilde{b}_{W}\right)\right|$ & $\leq$ & 0.44 & 0.44 \\
\hline
\end{tabular}

\section{4. $W W H$ couplings}

Due to missing neutrinos in the final state here one can only construct two observables: cross-section and forward-backward asymmetry with respect to polar angle of Higgs boson. Any deviation from SM value for cross-section largely depends on $\Delta a_{V}$ and $\Re\left(b_{V}\right)$ (CP even, $\tilde{\mathrm{T}}$ even). Similarly, FB asymmetry receives a large contribution from $\Im\left(\tilde{b}_{V}\right)$ (CP odd, $\tilde{\mathrm{T}}$ even). Hence there is no other direct observable to probe the remaining anomalous couplings. Assuming $\Delta a_{Z}=\Delta a_{W}=\Delta a$, we calculate the expressions for both the observables with $R 1$ and $R 2$ cuts. In table 3 we list the individual limits of sensitivity on the various anomalous couplings at $3 \sigma$ level. To see what the sensitivity will be when all the anomalous couplings were to be nonzero, we construct a nine-dimensional region in parameter space and take a point from that region and calculate all the observables simultaneously. If the difference from their SM values due to these anomalous couplings is within the statistical fluctuation in SM values of these observables, then we say that the point is inside the blind region. The points on the boundary of this region give us the simultaneous limit of sensitivity of these measurements to the anomalous couplings. These are listed in table 4 . These tables show that the lack of a specific observable to probe $\tilde{\mathrm{T}}$-odd couplings results in rather poor sensitivity to them. For more details, see [2]. 


\section{Conclusion}

We have analyzed the sensitivity of the process $e^{+} e^{-} \rightarrow f \bar{f} H, f$ being a light fermion and probe different anomalous couplings. We implement various kinematical cuts on the different final-state particles so as to reduce background and also take into account finite $b$-tagging efficiency. When these effects are removed, our analysis reproduces the results of [4]. Although the observables constructed using optimal observable analysis [3] have maximum sensitivity to the anomalous couplings, they are a little opaque to the physics that is being probed. The observables that we have constructed by taking expectation values of sign of the correlators are simple to construct and most of them have definite $\mathrm{CP}$ and $\tilde{\mathrm{T}}$ properties thus probing specific anomalous couplings. Apart from $\Re\left(b_{V}\right)$ and $\Delta a_{V}$, constraints on all the other anomalous couplings can be obtained using asymmetries and hence are robust to the effects of radiative corrections.

\section{References}

[1] See, for example, M Drees, R M Godbole and P Roy, Theory and phenomenology of sparticles (World Scientific, Singapore, 2004)

[1a] For detailed definition, see [2]

[2] Sudhansu S Biswal, Debajyoti Choudhury, Rohini M Godbole and Ritesh K Singh, Phys. Rev. D73, 035001 (2006)

[3] K Hagiwara, S Ishihara, J Kamoshita and B A Kniehl, Euro. Phys. J. C14, 457 (2000)

[4] T Han and J Jiang, Phys. Rev. D63, 096007 (2001) 